\title{
Medios sociales y democratización de la comunicación: del potencial emergente a los riesgos del presente
}

\section{Social media and the democratization of communication: from the emerging potential to the present risks}

\author{
Daniel Javier de la Garza Montemayor
}

Recepción: 31-05-2020

Aceptación: 09-07-2020

\section{Resumen}

En el presente artículo realizaremos un recuento de los acontecimientos más importantes que permitieron consolidar la percepción en torno a los medios digitales como una nueva modalidad de implicación cívica. Señalaremos una diferenciación de tres etapas en las que se consolidó la ciberpolítica: la primera de ellas, fue el potencial democratizador que inicia en 2008 con la campaña política de Obama, la Primavera Árabe y movimientos cívico-digitales en España, Chile y México. La segunda etapa fue aquella en la que se normalizan estas herramientas dentro de la competencia política y comunicación institucional, que transita del 2013 al 2018, año en que se celebran procesos electorales inéditos en Gran Bretaña y Estados Unidos. A partir de 2019, se inicia una tercera etapa en la que surgen nuevas interrogantes en torno al rol de los medios sociales en la consolidación de la democracia en distintas regiones del mundo.

Palabras clave: Internet, Redes Sociales, Ciberpolítica, Procesos Electorales, Transición.

\section{Abstract}

This article reflects on the most important events that allowed to consolidate the perception around digital media as a new form of civic involvement. In the text, we make a differentiation of three stages in which cyberpolitics was consolidated: The first of them was the democratizing potential, which began in 2008 with the Obama political campaign, the Arab Spring and civic-digital movements in Spain, Chile and Mexico. The next stage is that in which these tools are normalized within the political competition and institutional communication, which goes from 2013 to 2018, the year in which unprecedented electoral processes are held in Great Britain and the United States. Starting in 2019, we begin a third stage in which new questions arise about the role of social media in the consolidation of democracy in different regions of the world.

Key words: Social Networks, Cyberpolitics, Electoral Processes, Transition. 


\section{Introducción}

$7 \mathrm{n}$ el presente artículo, se llevará a cabo un repaso de las diferentes $\checkmark$ etapas en torno al auge, promesa, y finalmente escepticismo del potencial democratizador de los medios sociales en el ámbito público. Una vez que se ha cumplido una década desde que las noticias reveladas en el ciberespacio contribuyeron a generar una expectativa de democratización en la transmisión de la comunicación en una primera instancia, pero también existió una razonable expectativa sobre una mayor apertura gradual en la vida pública.

Se toma en consideración como marco de referencia temporal, doce años en los que la ciberpolítica terminaría por consolidarse, en parte, por los cambios sustantivos tecnológicos que lo harían posible, pero también por el debilitamiento progresivo de algunas estructuras tradicionales de poder político. Se pretende generar un registro que considere algunas de las expectativas que se despertaron durante este proceso, pero además, abrir una discusión en torno a los retos que se tienen en el presente.

El texto parte de dos antecedentes importantes: la campaña de Barack Obama en 2008 y el surgimiento de Wikileaks en 2010. Posteriormente, se analizan los efectos de la Primavera Árabe, el movimiento estudiantil conocido como el Invierno Chileno, la movilización del \#15-M en España y la irrupción del \#YoSoy132 en México. También se realiza una examinación del referéndum celebrado en Reino Unido y la elección presidencial de Estados Unidos de 2016, así como el papel que desempeñaron las redes sociales virtuales en cada uno de esos procesos electorales. Concluimos con una reflexión en torno a los retos del presente, cuando nos enfrentamos a la era de las percepciones y las noticias falsas.

\section{Las primeras manifestaciones}

\section{La elección de Barack Obama}

Existe un consenso en torno a que los medios sociales tuvieron su primera manifestación política importante durante la campaña de Barack 
Obama a la presidencia de los Estados Unidos de América en el año 2008. Cuando se celebró el proceso electoral, la popular red social Facebook cumplía con poco más de cuatro años de existencia y se extendía con una inusitada velocidad entre los más jóvenes (Kushin y Yamamoto, 2010).

La campaña de Barack Obama desde un inicio requería establecer una imagen innovadora con respecto a sus competidores. En una primera instancia, sorprendió durante la contienda interna del Partido Demócrata, cuando terminó por ser un reto serio frente a la Senadora Hillary Clinton, a quien la mayoría de las analistas consideraban como favorita para obtener la nominación (Bowden, 2010).

Es importante considerar que en los procesos electorales estadounidenses los votantes deben registrarse de manera previa a la elección, para poder ejercer su derecho al voto. Lo que significa que los competidores de dicha elección deben realizar un esfuerzo por persuadir a sus simpatizantes a inscribirse primero, y después, el día del proceso, a votar. Las redes sociales probaron ser un vehículo importante en la campaña de Barack Obama para lograr ambas tareas (Soní-Soto, 2011).

Por otra parte, existe el tema del financiamiento: En aquella elección se podía optar tanto a la subvención pública, como a la privada. En este caso, la campaña demócrata eligió el financiamiento privado, mientras que el candidato republicano John McCain se inclinó por el financiamiento público. También los medios sociales virtuales resultaron importantes para obtener una recaudación por parte de los simpatizantes, incluso cuando la movilización tradicional tuvo un enorme peso en la contienda (Toal, 2009).

El proceso electoral en la democracia más longeva del mundo se convertiría en el primer caso de estudio que demostraría el potencial de las redes sociales. Pero a pesar de la promesa, su actuación sería limitada en elecciones que se celebraron poco tiempo después: de acuerdo a Wring y Ward (2010), las redes sociales tuvieron poca incidencia en la conversación ciudadana en torno a la elección general en el Reino Unido. 


\section{Wikileaks y la ciberpolitica}

Dos años después de la creación de Facebook, emergió otro portal en Internet que terminaría siendo un tema de referencia durante los siguientes años; la plataforma Wikileaks, que abrió en el año 2006, con el fin de proteger las fuentes de filtraciones sobre noticias que pudieran incomodar al poder político. El sitio se encargaría de difundir aquellas noticias que expusieran abusos de poder, en un intento por exponer la corrupción que los medios tradicionales en muchos ocasiones silenciaban, lo que convirtió a ese medio en un fenómeno global (Quian y Elías, 2018).

En relación a lo anterior, es importante mencionar que existen diferencias entre los conceptos cibercrimen, ciberactivismo y la ciberguerra, que suelen confundirse por la opinión pública. En el primer caso, se trata de la obtención y utilización de datos personales mediante la vulneración de alguna cuenta virtual (Cai et al., 2018). En el segundo caso, el ciberactivismo se refiere de la organización social que se articula a través de medios digitales para impulsar causas, oponerse o aportar ideas (Sierra-Caballero, 2018). Y por último, en la ciberguerra hablamos de un campo de batalla moderno, en el que las principales armas son las herramientas tecnológicas que desplaza la forma tradicional de combate entre las principales potencias del planeta.

Desde la perspectiva de Hampson (2012), el sitio de Wikileaks correspondería a una categoría distinta debido a que la organización poseía una agenda política que algunos denominan como hacktivismo, refiriéndose a la utilización de herramientas digitales con medios tanto legales como ilegales, con el fin de incidir políticamente en una causa determinada.

Durante unos años, la plataforma Wikileaks, y su fundador, Julian Assange, tuvieron un éxito moderado al impulsar su agenda de transparencia. Existieron casos específicos donde las denuncias que se generaron en el sitio de Internet tuvieron una incidencia fuera del mundo virtual (Marechal, 2014).

En el año 2010, Wikileaks finalmente concentraría la atención de la opinión pública a nivel internacional. Durante este año, la plataforma recibió lo que fue considerado en su momento la mayor filtración de la historia, que 
a muchos recordó el caso de Daniel Ellsberg con los papeles del pentágono durante la Guerra de Vietnam (Ellsberg, 2002).

Se trataría de dos filtraciones mayores que fueron proporcionadas por la analista militar Chelsea Manning: donde salieron a la luz documentos sobre la guerra estadounidense en Afganistán y más de 250,000 cables diplomáticos de las principales embajadas de los Estados Unidos. En el primero se evidenciaban excesos durante el conflicto bélico, mientras que en el segundo se señalaba el injerencismo estadounidense en los asuntos de otras naciones (Curtis et al., 2015).

La información que se reveló durante esta crisis era tan amplia y abrumadora, que algunos medios tradicionales acordaron con Julian Assange su paulatina divulgación. Sin embargo, la reacción de Estados Unidos frente a lo que argumentaba era una vulneración mayúscula a la seguridad nacional, fue crear un cerco internacional a la plataforma de Wikileaks (Curran y Gibson, 2013).

El caso de Wikileaks iniciaría un debate importante por las siguientes razones: en primera instancia, aun cuando varios medios masivos dieron a conocer contenidos que provenían de la filtración, la fuente original era de un medio no convencional. Por otra parte, se generó un debate en torno a los límites de la información que debía hacerse del conocimiento público. En contraparte, también se argumentó que las plataformas digitales podrían hacer del conocimiento público asuntos gubernamentales que de otra forma se habrían mantenido reservados (Christensen, 2016).

Las revelaciones de Wikileaks tendrían consecuencias inmediatas, como fue su indicencia en la llamada Primavera Árabe. La información contenida en los cables diplomáticos exhibía no sólo la intervención estadounidense en otros países, también demostraba la corrupción de regímenes aliados (Mabon, 2013). 


\section{La era del potencial democratizador}

2011: Primavera Árabe: la revolución que emergió de las redes.

En los primeros meses de 2011, los ciudadanos de Túnez y Egipto se manifestaron de forma masiva en contra de sus gobernantes, logrando derrocarlos en cuestión de semanas, llegando a ser un acontecimiento tan impresionante como inesperado: En una región en donde parecía improbable la interrupción de dictaduras personales que habían durado décadas en el poder, de manera inusitada terminarían por mostrar una enorme vulnerabilidad (Soengas, 2013).

Se debe destacar que la visión sobre estos acontecimientos ha cambiado con el paso del tiempo. Los países antes mencionados no serían los únicos en los que se daría un alzamiento: en Siria y Libia diversos grupos sociales también se confrontaron con la clase gobernante. Pero a diferencia del caso de Túnez y Egipto, la colisión derivó en una sangrienta guerra civil en ambas naciones. De acuerdo con Portada y Billet (2019), la diferencia en los resultados radicó en las redes internacionales que terminarían por sostener a unos gobiernos, mientras que otros no contaron con apoyos similares.

Sin embargo, el alzamiento en la región fue consecuencia de la violación sistemática de los derechos humanos y la ausencia de las libertades políticas más elementales. También se trató de una dinámica en la que la frustración colectiva de un país se trasladó a otra con una rapidez inusitada, en lo que puede observarse un efecto dominó (Asfandyar y Ullah, 2019).

Una de las principales características de estas movilizaciones, fue el rol que desempeñó el Internet. De acuerdo con Lynch (2015), los medios electrónicos dieron voz a aquellos que no encontraban representación en los medios tradicionales. También los medios sociales funcionaron como un medio virtual, cohesionando a quienes se encontraban agraviados por el autoritarismo, para eventualmente salir a manifestarse a las calles.

En la perspectiva de Rennick (2013), las redes sociales resultaron fundamentales para organizar las protestas en contra de las autoridades durante este periodo, pero además, los actores que se agruparon de una 
manera tradicional tuvieron un efecto importante. En otras palabras, durante la Primavera Árabe el rol de los medios virtuales fue clave, pero sin la movilización popular y la resistencia que se había creado fuera del mundo virtual, el desenlace no habría sido el mismo.

A diferencia de otros acontecimientos recientes, los medios sociales sirvieron como un medio de adhesión, pero también como una fuente de difusión de un evento histórico. Muchos ciudadanos que no participaron en las manifestaciones, o que se encontraban lejos de su país de origen, pudieron seguir el desarrollo de las protestas a través de las plataformas digitales (Dalhgren, 2011).

A diferencia de otros acontecimientos históricos, los sucesos de la primera parte de 2011 fueron reconocidos muy pronto como una nueva modalidad de movilización social. Entre otras cosas, se auguraba que esta nueva generación de movimientos sociales se concentraría en generar cambios globales, más que demandas específicas (Glasius y Pleyers, 2013).

La visión global sobre la Primavera Árabe era en términos generales positiva. La mayoría de las reflexiones parten de valorar la proeza histórica, más que en las consecuencias finales de estos movimientos. Según ese momento, existía un optimismo razonable en torno a los efectos democratizadores de los medios sociales.

\section{\#15-M: De las redes a las calles}

En la primera parte de 2011, España sufría las consecuencias de la crisis global de 2008. La transición a la democracia había generado un sistema político estable donde se producía una alternancia ordenada en el poder, tanto a nivel nacional como en las distintas regiones del país. Pero la crisis económica terminaría por generar la percepción de una falta de capacidad de respuesta de las autoridades, por lo que una parte de los españoles dejó de sentirse representado (Castells, 2012).

El Movimiento \#15-M surge como una reacción al malestar de amplios actores sociales. También fue el punto de partida de otros movimientos similares, que, en este caso, se generaban a diferencia de la Primavera Árabe 
en países con sistemas democráticos. Este movimiento tendría repercusiones en el sistema político español, pero de acuerdo con Bonet i Martí (2015), el resultado resultó inconstante.

Una de las principales demandas del movimiento era la defensa de una auténtica democracia, que muchos sentían que había sido vulnerada por los representantes de los distintos partidos políticos. Además, se buscaba el rescate político tanto de los servicios que proporciona el estado, como espacios de incidencia que eran considerados como limitados (Espinar Merino, 2015).

El Movimiento \#15-M confirmó una tendencia sobre una nueva modalidad de movimientos sociales que se materializaban tanto de manera colectiva, como en la intimidad de espacios personales, las tecnologías de la información y la comunicación. Ante esto se generó un proceso de identificación donde, si bien, el propósito del movimiento era lograr un cambio en el sistema político español, la utilización del Internet como herramienta clave podría generar ganancias económicas para quienes comercializaban con sitios web, algo que el movimiento buscó combatir (Robles y CórdobaHernández, 2018). Esto último es importante, debido a que se registra uno de los primeros cuestionamientos sobre plataformas digitales que eran consideradas como espacios de mayor libertad, en comparación a los medios tradicionales de comunicación.

Fue un proceso donde se pudo diferenciar el registro que hicieron los medios tradicionales sobre el Movimiento \#15-M de aquel que realizaron los medios digitales emergentes, según las conclusiones de la investigación de García-Arranz (2014). Si bien, los medios convencionales reflejaron algunas opiniones de los ciudadanos, quienes tuvieron la última palabra fueron los periodistas. El registro de los sucesos que realizaron los medios digitales fue a la vez una fuente de información, como la difusión de opiniones ciudadanas diversas y distintas voces que pretendían proporcionar una explicación a los acontecimientos.

El movimiento social del \#15-M fue esencial para posicionar algunos temas dentro de la agenda pública desde los medios digitales. Fueron 
importantes las variadas y muy diversas demandas sociales que surgieron durante este movimiento, logrando al final que la mayoría de estas tuviese visibilidad en los medios de comunicación masiva (Casas, Davesa y Congosto, 2016).

Se discute en gran medida cuáles fueron las auténticas repercusiones del \#15-M en la vida pública española. En algunos casos, se considera que se trató de un punto de partida para una ciudadanía civil organizada (Sampedro y Lobera, 2014). Pero tal como habíamos mencionado antes, existen otras perspectivas que consideran que sus resultados fueron irregulares (Bonet $\mathrm{i}$ Martí, 2015).

No obstante, el \#15-M fue un movimiento político de vanguardia, que independiente de los resultados, sería clave en trasladar la deliberación que se producía en las redes sociales a las calles. Este mismo proceso se repetiría (aunque enarbolando causas distintas y en contextos políticos diferentes) en otros dos países iberoamericanos durante los siguientes meses.

Invierno Chileno: de la protesta estudiantil a la insurrección generalizada.

En el caso de Chile, surgirían protestas por parte del sector estudiantil en contra del modelo educativo que prevalecía en ese país. Es importante tomar en consideración que en este país habían transcurrido 21 años desde que el dictador Augusto Pinochet abandonó el poder. Sin embargo, el modelo económico y educacional de la dictadura tuvo pocas alteraciones desde entonces (Slachevsky Aguilera, 2015).

Una de las principales herencias del régimen de Pinochet fue el control mayoritariamente del sector privado sobre la educación. Esto generaba como consecuencia que los costos de cursar una carrera universitaria resultaran demasiado onerosos para un amplio sector de la población, por lo que muchos de ellos recurrían al endeudamiento para poder tener acceso a una carrera profesional (Donoso, 2013).

En el mes de abril de 2011, estudiantes de distintas universidades terminarían por manifestarse dentro y fuera de los medios sociales, con el fin 
de demandar un sistema educativo distinto. Este movimiento se extendería con fuerza hasta el mes de agosto de ese año, lo que le permitió posicionar en el imaginario colectivo la demanda de un mayor acceso a una educación profesional (Eltit, 2012).

Al igual que ocurrió con el Movimiento \#15-M, en Chile el uso de los medios sociales resultó fundamental para activar la movilización social. Desde entonces se advertía que dada la alta conectividad que prevalecía en ese país a principios de la década pasada, redes sociales como Facebook podrían ser clave para atraer a los jóvenes a participar en asuntos de la vida pública (Scherman y Arriagada, 2011).

De acuerdo con un estudio de Valenzuela, Arriagada y Scherman (2012), existían algunos factores que podían predecir la predisposición a realizar protestas, por ejemplo "agravios políticos, orientación izquierdista, interés en política y participación en grupos cívicos" (p.311). Por otra parte, el estudio también mencionaba que las redes sociales como Facebook, más que crear nuevas maneras de implicación, potencializaban las formas existentes.

Poco tiempo después de las protestas generadas durante 2011, la juventud chilena comenzaba a informarse sobre asuntos de la vida pública, principalmente a través de las redes sociales. En un estudio de Condeza, Bachmann y Mujica (2014), fue posible concluir que la oferta de los medios de comunicación masiva había dejado de ser atractiva para aquellos jóvenes que se encontraban entre los 13 y los 17 años.

La tendencia que inició durante las protestas generadas en esta etapa terminaría por consolidarse durante las elecciones en Chile de 2013. Según la indagación de Bacallo-Pino (2016), las redes sociales fueron un espacio fundamental para que el movimiento continuara posicionando sus demandas en la agenda electoral

Es posible argumentar que en las protestas que cimbraron a Chile a finales de 2019, se afianzó la tendencia de utilizar los medios sociales como un canal de organización para articular la movilización. A diferencia de lo acontecido en 2011, la convocatoria en redes sociales no se limitó a los jóvenes, pues un amplio sector de la población salió a las calles para reclamar 
cambios sustantivos al modelo económico y a modificar o crear una nueva constitución del Estado Chileno (Pizarro Hofer, 2020).

\#YoSoy132: El principio del fin de la hegemonía.

En comparación con el caso de Chile, en México, la movilización juvenil articulada por medios sociales se realizó en una coyuntura electoral. En este país, los procesos electorales para renovar al titular del Ejecutivo Federal se realizan con una periodicidad de seis años, el más largo en el continente latinoamericano, pero sin posibilidad de optar por la reelección.

La sociedad mexicana había padecido una larga temporada de violencia durante los gobiernos de Vicente Fox Quesada (2000-2006) y Felipe Calderón Hinojosa (2006-2012). Como consecuencia del desgaste de dos administraciones federales consecutivas del Partido Acción Nacional (PAN) y el temor que generaba en ciertos sectores la posible llegada del aspirante de izquierda Andrés Manuel López Obrador, las encuestas auguraban un posible regreso del Partido Revolucionario Institucional (PRI) al poder, que había gobernado a México 71 años de manera consecutiva antes del triunfo de Vicente Fox (De la Garza y Barredo, 2017).

El aspirante con más ventaja dentro del PRI era el entonces gobernador del Estado de México, Enrique Peña Nieto. El joven político aparecía con frecuencia en televisión, e incluso contrajo nupcias con una popular actriz de telenovelas. Desde el inicio de su administración, un trabajo periodístico había denunciado la existencia de un acuerdo comercial entre la televisora más importante del país (Televisa) y el político prísta. Este convenio de publicidad consistía en un gasto importante con cargo al presupuesto del Estado de México en la televisora, que, a su vez, garantizaría una cobertura favorable que apoyara las aspiraciones presidenciales de Peña Nieto (De la Garza y Barredo, 2018).

Enrique Peña Nieto se presentó al proceso electoral como favorito, y su triunfo se asumía como inevitable en casi todos los sectores. La campaña electoral en México de 2012 tuvo una duración de tres meses, y durante las primeras semanas, no existió algún acontecimiento que contribuyera a alterar la percepción colectiva sobre su triunfo inevitable. Pero el 11 de mayo, la 
narrativa del proceso electoral habría de cambiar de manera permanente (Nabel, 2015).

Durante una visita a una universidad privada mexicana, el candidato Peña Nieto fue increpado por los estudiantes, y su fría respuesta a los cuestionamientos causó malestar y un rechazo inmediato. Las reacciones por parte de los jóvenes fueron grabadas y compartidas en redes sociales, teniendo una enorme difusión. Sin embargo, Televisa, a quien habían acusado de mantener un contubernio con Peña Nieto para facilitar su llegada al poder, lejos de registrar los acontecimientos de forma veraz, dio voz a los operadores de la campaña del PRI quienes incluso cuestionaron que aquellos que increparon a Peña Nieto eran realmente estudiantes o no (Galindo y González, 2013).

Posteriormente y como respuesta, mediante un video, 131 jóvenes se identificaron como estudiantes de la universidad. Por lo que surgió entonces, en medios sociales, el movimiento denominado \#YoSoy132, que convocó a los estudiantes a manifestarse en las calles para demostrar su rechazo a la candidatura de Enrique Peña Nieto.

Este movimiento tuvo un crecimiento inesperado en pocos días y de muchas maneras, contribuyó a cambiar la conversación de una elección presidencial que los principales grupos de poder económico y mediático esperaban que se produjera sin mayores incidentes. El movimiento estudiantil se proponía evitar la victoria de Enrique Peña Nieto en la elección presidencial, pero también propugnó por la democratización de los medios tradicionales de comunicación (Portillo, 2015).

De la misma forma en que la movilización juvenil sorprendió por su crecimiento vertiginoso, tuvo una caída dramática después de que se celebró el proceso electoral. En particular, el movimiento no había conseguido cumplir con el propósito de impedir la victoria de Peña Nieto en las urnas, por lo que muchos en aquel momento dudaron de la eficacia no sólo de la movilización juvenil; sino que se cuestionó el alcance real de los medios sociales. Sin embargo, en 2012, se generó un cisma apenas perceptible para muchos mexicanos: Desde ese año comenzó un declive en la credibilidad de 
los medios de comunicación masiva. Seis años después, Televisa tuvo una incidencia mucho más reducida en la elección presidencial de 2018 (de la Garza y Pineda Rasgado, 2018).

\section{La expectativa democratizadora y la normalización de las herramientas digitales.}

Una suave y paulatina transición: de la comunicación politica digital a la consolidación de percepciones.

Aun cuando no se materializarán cambios políticos de manera inmediata, la percepción de muchos analistas y académicos sobre el potencial democratizador de los medios sociales era positiva. En términos generales, el debate en aquellos momentos se centraba en la manera en que incidían los medios digitales, tanto en el debate político, pero también como un medio de implicación (Vesnic-Alujevic, 2012; Red, 2013).

En el caso de los países de Latinoamérica, prevalecía una brecha digital mayor a la que existía en tiempo presente. Por esta razón se daba un escepticismo en torno a la posibilidad que tenían las redes sociales para desplazar a los medios tradicionales como fuentes principales de información (De la Garza y Barredo, 2017).

También fue evidente para diversas instituciones, tanto públicas como privadas, que la manera de recibir información estaba cambiando, al menos en lo que concernía a los jóvenes. Por esa razón, existió un incremento sustantivo en la utilización de los medios sociales durante esta época y de manera expedita, surgió una oferta cada vez más amplia de presuntos expertos que argumentaban que podían potencializar los efectos de las redes sociales con propósitos comerciales o electorales.

Ahora bien, esta relación generó un interés en el crecimiento de la academia a nivel global. Se realizaron estudios tanto cuantitativos como cualitativos en diversos contextos y en diferentes momentos históricos. En resumen, los resultados de los estudios parecían no coincidir demasiado más que en un eje en particular: la validez de los resultados de estudios sobre los efectos de los medios sociales en la participación política de los ciudadanos 
era pertinente para un momento y entorno determinado, como señalaba el estudio de Bimber y Copeland (2013)

De igual manera, diversas investigaciones registraron que la información en redes sociales sobre asuntos públicos comenzaba a ser consultada con la misma frecuencia (y en varios casos aún más) que las noticias de los medios convencionales. En estos años se da una transición que permite visualizar la asimilación de las nuevas tecnologías de la información y la comunicación a la estrategia de mercadotecnia de empresas, a la comunicación social de las instituciones públicas, y como un medio indispensable en la disputa política.

\section{Brexit, la mayoría invisible.}

Los resultados del plebiscito celebrado en las elecciones de Gran Bretaña de 2016 terminarían por sorprender al mundo entero. Con una mayoría ajustada, los electores del Reino Unido decidieron salir de la Unión Europea (UE). Después de la crisis económica griega que tuvo su punto máximo en el año 2015, se consideraba que la UE comenzaría a fragmentarse con la salida de la nación helena. Un año después, se materializaba una ruptura, pero de parte de una de las naciones económicamente más poderosas de la Unión.

Hacia el exterior, la elección para decidir si el Reino Unidos seguiría o no dentro de la Unión Europea, parecía hasta cierto grado predecible. Pocos observadores internacionales consideraban que el Brexit tenía serias posibilidades de prosperar. Más bien parecía que el referéndum correspondía a una necesidad del entonces primer ministro David Cameron de cumplir con los acuerdos que le permitieron formar un segundo gobierno (Cameron, 2019).

Cuando se convocó el plebiscito para consultar a la ciudadanía del Reino Unido si seguían o no en la UE, se conformaron dos bloques electorales: Remain (Permanecer) y Leave (Salida). El apoyo a los bloques no tuvo disciplina partidista: los principales partidos de Reino Unido (Conservador y Laborista) que se dividieron, y por lo que era común encontrar a partidarios de ambas instituciones apoyar las opciones del Remain o Leave, según cual fuese su convicción política. Pero también existieron grupos nacionalistas 
que vieron en la campaña por la salida, una posibilidad de reivindicación de su agenda política (Oliver, 2017).

Cabe notar que los partidarios de la salida del Reino Unido realizaron una campaña que se basó en encontrar a aquellos electores que se hallaban distanciados del sistema político. En otras palabras, aquellos ciudadanos que no se sentían representados por los partidos políticos tradicionales. Una parte importante de quienes habían dejado de sufragar eran los que pertenecían a la clase media que había visto su poder adquisitivo mermado de manera progresiva durante los últimos años (Johnstona et al., 2018).

Este segmento del electorado tenía nostalgia del antiguo esplendor imperial británico y desconfiaban de los inmigrantes. Como respuesta a la desafección que detectaron en ese sector, crearon un slogan que los motivaría a movilizarse el día del referéndum: Take Back Control (Tomar de nuevo el control). En esencia, la campaña a favor de la salida buscaba estimular tanto el sentimiento patriótico británico, como el rechazo a la presunta sangría económica que les representaba el pertenecer a la UE (Mancosu y Bobba, 2019).

En ese momento, los medios sociales resultaron ser un canal indispensable para activar a aquellos ciudadanos que de otra manera quizás se habrían abstenido de votar. Es posible argumentar que las encuestas tradicionales no terminaron por registrar el voto oculto a favor de la salida. El equipo de campaña a favor del Brexit había identificado que la elección podría ganarse si podía persuadir que el voto a favor de la salida representaba un rechazo en contra del sistema político en su conjunto (Shipman, 2016).

Es necesario resaltar que la campaña a favor de la permanencia se basó principalmente en una estrategia convencional, con un mensaje basado en la racionalidad y una fuerte apuesta a los medios convencionales. Mientras que la campaña a favor de la salida se centraría en lograr lo contrario: más que apelar a los hechos, buscó principalmente dirigirse a las emociones de los espectadores por medio de las redes sociales (Clarke y Newman, 2017).

La campaña del referéndum en Reino Unido de 2015 fue importante no sólo por la utilización de los medios sociales en la campaña electoral, sino 
también por la manera en que lo hizo. En principio, los que abogaron por la salida tuvieron la posibilidad de dirigirse de manera directa al segmento del electorado que compartía las mismas frustraciones. Por otra parte, lograron ser la campaña disruptiva dentro de la contienda. Unos cuantos meses después, el mundo atestiguaría una sorpresa similar durante la elección presidencial en Estados Unidos durante 2016 (Hall et al., 2018).

\section{Estados Unidos: La victoria de Donald Trump}

Desde que comenzaron a celebrarse las primarias durante el proceso electoral estadounidense, fue evidente que este era un proceso atípico. Contra todo pronóstico, el conocido magnate Donald Trump emergía como un poderoso contendiente frente a los demás aspirantes del Partido Republicano (Cay, 2017).

Desde un principio, la postulación de Donald Trump había sido cuestionada por sus declaraciones xenófobas, pero también había sido menospreciada por muchos que consideraban que no tenía posibilidades serias de conseguir la nominación como candidato. Pero existía un terreno en el cual el aspirante Trump sabía conducirse con una peculiar facilidad, que era el de los medios sociales (Jackson, 2018).

Donald Trump era reconocido no sólo por su personalidad extrovertida, declaraciones controvertidas y una tendencia a volver los asuntos públicos en un espectáculo. También tenía la habilidad de trasladar los aspectos más controvertidos de su discurso político a las redes sociales. Sus declaraciones causaban una considerable polémica, pero también la adhesión de grupos sociales que lo veían con admiración (Ghazal Aswad, 2019).

En el caso de la contienda por la nominación del Partido Demócrata, también existieron claras señales que apuntaban a un cambio sustantivo en una parte del electorado. Bernie Sanders, un candidato que se autodenominaba socialista, terminó por ser un competidor importante en una contienda en la que se esperaba que la ex Secretaria de Estado, Hillary Clinton, ganara con facilidad (Sanders, 2018). 
El discurso de Bernie Sanders recordaba a las demandas sociales de aquellos que se organizaron alrededor del movimiento \#OccupyWallStreet. En esta movilización, las redes sociales fueron un punto de encuentro fundamental para los grupos sociales que se encontraban inconformes con la desigualdad económica que prevalecía en su país. El movimiento tuvo una importante repercusión en el debate público estadounidense, aun cuando no se materializaron medidas concretas a partir de sus demandas (Hill, 2011).

La campaña de Sanders estuvo dirigida a aquellos electores que consideraban que el Partido Demócrata había dejado de representar a la clase trabajadora. Cabe destacar que las redes sociales fueron fundamentales para difundir los contenidos del candidato y también para recaudar fondos. Por otro lado, Hillary Clinton apostó más por los medios tradicionales, de los que obtuvo un apoyo casi unánime (Allen y Parnes, 2018).

Una vez que Clinton y Trump fueron nombrados los contendientes del Partido Demócrata y el Partido Republicano, respectivamente, se desarrolló una de las elecciones más ríspidas en la historia de Estados Unidos. Ambos candidatos se atacaron con severidad, utilizando todas las herramientas que tenían a su disposición. En términos de comunicación política, al igual que en el caso del referéndum en Gran Bretaña, existió un notable contraste entre las estrategias de los dos aspirantes. Mientras que Donald Trump apostó de manera decidida a las redes sociales, Hillary Clinton basó su estrategia principalmente en los medios convencionales, como se mencionó antes (Wead, 2017).

El resultado de la elección fue imprevisible: aun cuando recibió menos votos que Hillary Clinton, Donald Trump ganó la elección presidencial en el colegio electoral. Las posteriores revelaciones en torno al caso de Cambridge Analytics, representarían una fuerte controversia sobre la utilización de información personal con fines políticos, como lo registra de manera puntual Kaiser (2019).

Para el año 2016, las redes sociales habían dejado de ser una novedad, resultaban indispensables para cualquier tipo de estrategia electoral, comunicación institucional e inclusive eran un medio indispensable para 
la comercialización de productos y servicios. Pero el debate comenzaba a ser otro, uno que cuestionaría el real aporte de las redes al debate público y la fabricación creciente de noticias falsas que terminaban por confundir y polarizar a la opinión pública (Walters, 2018).

\section{La era de la incertidumbre}

A principios de 2010, se consideraba que las redes sociales podrían burlar la censura en regímenes autoritarios que controlaban los medios tradicionales. Lo cierto es que, en la actualidad, los medios sociales virtuales coexisten con varios sistemas que están lejos de poder ser considerados democráticos, como lo destaca Zeynep (2018).

Resulta alarmante que las plataformas virtuales han terminado por ser el vehículo de algunos grupos extremistas, como menciona Marantz (2019), que terminan por esparcir odio entre los usuarios. El potencial de deliberación entre los internautas, salvo en casos muy emblemáticos, ha sido reducido.

En términos de comunicación política, las redes sociales pueden ser el canal ideal para la demagogia y el fanatismo. Un caso emblemático de esto fue la elección de Jair Bolsonaro en el Brasil. Este político era conocido durante su campaña por sus frases misógenas, homofóbicas y rascistas. En un estudio de Canavilhas et al., (2019) se destaca que expresiones de odio fueron compartidas o mimetizadas por sus simpatizantes y se volvieron emblemáticas de la contienda electoral

Uno de los aspectos de mayor riesgo en este proceso fue el uso de información privada por parte de gobiernos, partidos políticos y los mismos administradores de medios sociales. El caso que se mencionó de manera previa, Cambridge Analytics, demostró que los datos de los usuarios de Facebook fueron utilizados sin su consentimiento para contribuir a generar estrategias de campaña de distintos países que contrataron los servicios de la empresa (Heawood, 2018).

Cabe mencionar que el espionaje a los usuarios a través de las plataformas digitales había sido denunciado desde 2013 por Edward Snowden (2019), pero la advertencia comenzó a ser ignorada con el paso del 
tiempo. Lo cierto es que desde el inicio de las redes sociales virtuales, los usuarios utilizan estos medios para comunicarse (mediante texto, imágenes, audio y video), expresar sus opiniones, compartir contenido, establecer conexiones con otros y consultar información de empresas, celebridades, figuras públicas, partidos políticos, organizaciones de la sociedad civil, entre otras acciones que permiten realizar una radiografía sobre un internauta en particular, pero que a su vez nos puede proporcionar una visión panorámica de las preferencias y expresiones ciudadanas con una precisión con la que pocos estudios de mercado habrían contado en el pasado.

Otro aspecto que resulta tan irónico como preocupante, es la desinformación que se genera a tráves de estas herramientas virtuales. En momentos donde es posible transmitir información como nunca antes en la historia, la veracidad de la misma tambien resulta en muchos casos cuestionable.

A principios de la década de 2010, se consideraba que las redes sociales virtuales podrían ser un contrapeso ciudadano y periodístico al monopolio informativo que ejercían los medios masivos de comunicación. Pero desde entonces, Castells (2011) advertía la posibilidad de que aquella información que se compartiera en medios virtuales fuera originaria de los medios tradicionales, algo que se ha confirmado con el paso de los años.

Asimismo, existen noticias falsas generadas por usuarios (célebremente denominadas fake news), que al margen de una agenda política o económica que puedan tener, contribuyen a generar confusión en la opinión pública (Margetts, 2019). Fue precisamente la desinformación de los medios masivos de comunicación lo que contribuyó a generar una crisis de credibilidad que terminaría por afectarles en términos de audiencia (Natanson, 2014).

El potencial democratizador de los medios sociales es aún una posibilidad desde una perspectiva teórica, pero existe suficiente evidencia para argumentar que la ausencia de un clima de tolerancia en las democracias contemporáneas difícilmente pueda resarcirse con la existencia de los medios digitales.

En el ciberespacio se exhiben los grupos sociales que se rehusan 201 
a aceptar la pluralidad como una forma de vida. El debate estridente y la polarización estéril difícilmente contribuirán a generar un espacio de convivencia de intercambio y diálogo que las propias herramientas digitales tienen el potencial de generar.

Cabe destacar la responsabilidad por parte de agentes económicos, gobiernos y no pocas fuerzas políticas en la utilización deshonesta de los medios digitales como un espacio para generar adhesiones a causas particulares. En síntesis, la nueva década comienza con más interrogantes que respuestas en un proceso que ha cambiado y que quizás puede resultar irreversible.

\section{Conclusiones}

En el presente texto, hemos propuesto que en retrospectiva el fenómeno emergente de los medios sociales, como medios de artículación para la organización colectiva y la implicación cívica, se puede dividir en tres diferentes etapas generales. En un principio, existió un enorme entusiasmo por parte de un sector importante de la opinión pública y la academia en torno a las posibilidades que brindaban las entonces nuevas tecnologías para contrarrestar el poder hegemónico de los medios masivos de comunicación, así como un canal de comunicación horizontal entre ciudadanos.

En este apartado, tanto la Primavera Árabe en Túnez y Egipto como los movimientos que acontecieron en países iberoamericanos como España, Chile y México entre 2011 y 2012, contribuyeron a reforzar la promesa de los medios sociales virtuales como agentes de cambio democratizadores. También existía la expectativa que estas herramientas contribuyeran a transparentar el ejercicio de gobierno y a fortalecer la rendición de cuentas.

En una segunda etapa, el carácter disruptivo de los medios sociales comenzó a asimilarse y se avanzó en la gestión profesional de estos medios por parte de organizaciones públicas y privadas. Este proceso terminaría por trasladar algunos de los principales debilidades de los medios tradicionales (como la desinformación) con algunas nuevas características: La publicidad podría ser dirigida a un segmeto de la población específico y en algunos casos incluso se utilizaría la información personal de los usuarios (sin su 
consentimiento) con el fin de elaborar mensajes más eficaces, como nunca antes en la historia se había visto.

Esta transición nos sitúa en la actual era de la incertidumbre, siendo innegable que las redes han funcionado como un espacio de encuentro entre ciudadanos que creen en una determinada causa y buscan contribuir a cambiar su entorno. Pero también resulta contundente argumentar que no han sido el espacio de deliberación pública que algunos auguraban; como muestra de ello se puede señalar que existe una alta exposición a noticias apócrifas. Es por esto que el paso del tiempo y futuras investigaciones podrán proporcionar más información en torno a si las redes tienen un efecto positivo en el fortalecimiento de distintas expresiones democráticas, o por el contrario, que se consolide una realidad en la que predomine la desinformación y la utilización de la tecnología como un medio que busque el control social. 


\section{Referencias}

Allen, J. y Parnes, A. (2018). Shattered: Inside Hillary Clinton's Doomed Campaign. New York City: Random House LCC US.

Asfandyar; F. y Ullah, M. (2018). “The Arab Spring Genesis: Implications for Global Politics”. Dialogue, 13 (4), 1-16.

Bacallao-Pino, L. M. (2016). "Redes sociales, acción colectiva y elecciones: los usos de Facebook por el movimiento estudiantil chileno durante la campaña electoral de 2013". Palabra Clave 19 (3), 810-837. doi: 10.5294/ pacla.2016.19.3.6.

Bimber, B. y Copeland, L. (2013). "Digital Media and Traditional Political Participation Over Time in the U.S". Journal of Information Technology \& Politics, 10 (2), 125-137. doi: 10.1080/19331681.2013.769925.

Bonet i Martí, J. (2015). "Movimiento del 15-M: La fuerza politizadora del anonimato". ACME: An International E-Journal for Critical Geographies, 14 (1), 104-123.

Bowden, G. (2010). "Obama, Palin, and Weber: Charisma and Social Change in the 2008 U.S. Election". Canadian Review of Sociology, 47 (2), 171-190. doi: 10.1111/j.1755-618X.2010.01229.x.

Cai, T., Du, L., Xin, Y. y Chang, L. (2018). "Characteristics of cybercrimes: evidence from Chinese judgment documents". Police Practice \& Research, 19 (6), 582-595. doi:10.1080/15614263.2018.1507895

Cameron. D. (2019). For the Record. New York: Harper

Canavilhas, J., Colussi, J. y Moura, Z. (2019). “Desinformación en las elecciones presidenciales 2018 en Brasil: un análisis de los grupos familiares en WhatsApp". El profesional de la información, 28 (5), e280503. doi:https://doi.org/10.3145/epi.2019.sep.03.

Casas, A., Davesa, F. y Congosto, M. (2016). "La cobertura mediática de una acción "conectiva": la interacción entre el movimiento 15-M y los me- 
dios de comunicación”. Revista Española de Investigaciones Sociológicas, (155), 73-96. doi: http://dx.doi.org/10.5477/cis/reis.155.73.

Castells, M. (2011). “A Network Theory of Power". International Journal of Communication, 5, 773-787.

Castells, M. (2012). Redes de Indignación y Esperanza. Madrid: Alianza Editorial.

Cay Johnston, D. (2017). The Making of Donald Trump. New York City: Random House LCC US.

Christensen, C. (2016). "The links that bind: WikiLeaks, Twitter, and the Julian Assange case". Popular Communication, 14 (4), 224-237. doi: https://doi.org/10.1080/15405702.2016.1234268.

Clarke, J. y Newman, J. (2017). "What's the subject? Brexit and politics as articulation”. Journal of Community \& Applied Social Psychology, 29 (1), 67-77. doi: 10.1002/casp.2376.

Condeza, R., Bachmann, I. y Mujica, C. (2014). "News Consumption among Chilean Adolescents: Interests, Motivations and Perceptions on the News Agenda". Comunicar, 22 (43), 55-64. doi: 10.3916/C43-2014-05.

Curran, G. y Gibson, M. (2013). "WikiLeaks, Anarchism and Technologies of Dissent”. Antipode, 45 (2), 294-314. doi: 10.1111/j.14678330.2012.01009.x.

Curtis, A., Ye, X., Hachey, K., Bourdeaux, M. y Norris, A. (2015). "A space-time analysis of the WikiLeaks Afghan War Diary: a resource for analyzing the conflict-health nexus". International Journal of Health Geographics, 14, 1-14. doi: 10.1186/s12942-015-0022-8.

Dahlgren, S. (2011). "What is a revolution? and other anthropological questions on the Arab Spring". Suomen Antropologi: Journal of the Finnish Anthropological Society, 36 (4), 73-76.

De la Garza, D. y Barredo, D. (2017). “Democracia digital en México: 
Un estudio sobre la participación de los jóvenes usuarios mexicanos durante las elecciones legislativas federales de 2015". Index Comunicacion, 7 (1), 95-114.

De la Garza, D. y Barredo, D. (2018). "Redes sociales, participación ciudadana y medios alternativos: la crisis de los medios masivos de comunicación en México durante el sexenio de Enrique Peña Nieto". En Zugasti, R., Mancho, A, y Segura, A. (Coords.). La opinión pública y la comunicación politica en entornos digitales (pp. 11-28). Zaragoza: Agregius Ediciones.

De la Garza, D. y Pineda Rasgado, M. (2018). “Consumo de medios y participación política de los jóvenes universitarios en el contexto electoral mexicano del 2018". Correspondencia \& Análisis, (8), 75- 95. doi: https:// doi.org/10.24265/cian.2018.n8.04

Donoso, S. (2013). "Dynamics of Change in Chile: Explaining the Emergence of the 2006 Pingüino Movement". Journal of Latin American Studies, 45 (1), 1-29. doi: 10.1017/S0022216X12001228.

Ellsberg, D. (2002). Secrets: A Memoir of Vietnam and the Pentagon Papers. Londres: Penguin Books.

Eltit, D. (2012). “Camila Vallejo: misión cumplida”. Debate Feminista, 46 (23), 189-198.

Espinar Merino, R. (2015). "El espacio público y la pugna por el significado de la Democracia. El debate alternativo sobre el Estado de la Nación en el movimiento 15-M". ACME: An International E-Journal for Critical Geographies, 14 (1),61-74.

Eun, Y. y Sita, J. (2016). "Cyberwar: Taking Stock of Security and Warfare in the Digital Age". International Studies Perspectives, 17 (3), 343360. doi: 10.1111/insp.12073.

Galindo, J. y González, J. (2013). \#Yosoy132: La Primera Erupción Visible. Distrito Federal: Global Talent University Press.

García-Arranz, A.M. Junio de 2014. "La opinión publicada sobre el 206 
movimiento 15M. Un análisis empírico de los periódicos digitales españoles: elmundo.es, elpais.com y abc.es". Palabra Clave , 17 (2), 320-352.

Ghazal Aswad, N. (2019). "Exploring Charismatic Leadership: A Comparative Analysis of the Rhetoric of Hillary Clinton and Donald Trump in the 2016 Presidential Election”. Presidential Studies Quarterly, 49 (1), 5674. doi: 10.1111/psq.12490.

Glasius, M. y Peleyers, G. (2013). "The Global Moment of 2011: Democracy, Social Justice and Dignity". Development and Change, 44 (3), 547-567. doi: 10.1111/dech.12034

Hall, W., Tinati, R. y Jennings, W. (2018). "From Brexit to Trump: Social Media's Role in Democracy". Computer, 51 (1), 18-27. doi: 10.1109/ MC.2018.1151005

Hampson, N. C. (2012). "Hacktivism: A new breed of protest in a networked world". Boston College International \& Comparative Law Review, 35 (2) 511-542.

Heawood, J. (2018). "Pseudo-public political speech: Democratic implications of the Cambridge Analytica scandal". Information Polity: The International Journal of Government \& Democracy in the Information Age, 23 (4), 429-434. doi:10.3233/IP-180009.

Hill, S. (2011). Digital Revolutions: Activism in the Internet Age. Seattle: WorldChanging.

Jackson Johnson, S. (2018). "Donald Trump, Disruptive Technologies, and Twitter's Role in the 2016 American Presidential Election". Widener Law Journal, 27 (1), 39-82.

Johnstona, R., Manley, D., Pattieb, C. y Jonesa, K. (2018). “Geographies of Brexit and its aftermath: voting in England at the 2016 referendum and the 2017 general election". Space and Polity, 22 (2), 162-187. doi: https://doi.org/10.1080/13562576.2018.1486349. 
Kaiser, B. (2019). Targeted: The Cambridge Analytica Whistleblower's Inside Story of How Big Data, Trump, and Facebook Broke Democracy and How It Can Happen Again. New York City: Harper.

Kushin, M. T. y Yamamoto, M. (2010). "Did Social Media Really Matter? College Students' Use of Online Media and Political Decision Making in the 2008 Election". Mass Communication \& Society, 13 (5), 608-630. doi:10.1080 /15205436.2010.516863

Lynch, M. (2015). "How the media trashed the transitions". Journal of Democracy, 26 (4), 90-99. doi: 10.1353/jod.2015.0070.

Mabon, S. (2013). “Aiding Revolution? Wikileaks, communication and the 'Arab Spring' in Egypt". Third World Quarterly, 34 (10),1843-1857. doi: http://dx.doi.org/10.1080/01436597.2013.851901

Mancosu, M. y Bobba, G. (2019). "Using deep-learning algorithms to derive basic characteristics of social media users: The Brexit campaign as a case study". PLOS ONE, 14 (1), e0211013.

Marantz, A. (2019). Antisocial: Online Extremists, Techno-Utopians, and the Hijacking of the American Conversation. New York City: Viking.

Marechal, N. (2014). "WikiLeaks and the Public Sphere: Dissent and Control in Cyberworld". International Journal of Technology, Knowledge \& Society: Annual Review, 9 (3), 93-106. 14. doi: 10.18848/1832-3669/cgp/ v09i03/56390.

Margetts, H. (2019). "Rethinking Democracy with Social Media”. Political Quarterly, 90, 107-123. doi: 10.1111/1467-923X.12574.

Nabel, L. T. (2015). “¿Quién programa las redes sociales en Internet? El caso de Twitter en el movimiento\# Yosoy132 México". Revista Internacional de Sociología, 73 (2), 63-75. doi:10.3989/ris.2013.05.29

Natanson, J. (2014). "La triple crisis de los medios de comunicación”. Nueva Sociedad, (249), 50-60. 
Oliver, C. (2017). Unleashing Demons: The Inside Story of Brexit. London: Hodder \& Stoughton.

Pizarro Hofer, R. (2020). “Chile: rebelión contra el Estado subsidiario". Trimestre Económico, 87 (346), 333-365. doi: 10.20430/ete.v87i346.1055.

Portada, R. y Billet, L. (2019). "The Finger on the Scale: International Interventions in the Middle East and North Africa Following the Arab Spring". Journal of Global South Studies, 36 (1), 23-58. doi: 10.1353/ gss.2019.0003.

Portillo, M. (2015). “Construcción de ciudadanía a partir del relato de jóvenes participantes del \#YoSoy132”. Global Media Journal México, 12, $1-18$.

Quian, A. y Elías, C. (2018). "Strategies and Reasons for the Impact of WikiLeaks on World Public Opinion". Revista Española de Investigaciones Sociológicas, (162), 91-110. doi:http://dx.doi.org/10.5477/cis/reis.162.91.

Red, M. (2013). “Rocking the Vote in Mexico's 2012 Presidential Election: Mexico's Popular Music Scene's Use of Social Media in a Post-Arab Spring Context”. International Journal of Communication, 7, 1205-1219.

Rennick, S. (2013). "Personal grievance sharing, frame alignment, and hybrid organizational structures: the role of social media in North Africa's 2011 uprisings". Journal of Contemporary African Studies, 31 (2), 156174. doi:10.1080/02589001.2013.781322.

Robles, J. M y Córboda-Hernández, A. M. (2018). “Commodification and digital political participation: The "15-M Movement" and the collectivization of the Internet”. Palabra Clave, 21 (4), 992-1022. doi: 10.5294/pacla.2018.21.4.3

Sampedro, V. y Lobera, J. (2014). “The Spanish 15-M Movement: a consensual dissent?” Journal of Spanish Cultural Studies, 15 (1/2), 61-80. doi: 10.1080/14636204.2014.938466.

Sanders, B. (2018). Our Revolution. New York City: Macmillan USA. Scherman, A. y Arriagada, A. (2012). “Jóvenes, disposición a votar 209 
y consumo de medios en Chile". Comunicación y Sociedad, 25 (1), 85-112.

Shipman, T. (2016). All Out War: The Full Story of How Brexit Sank Britain's Political Class. New York City: Harper Collins Publishers.

Sierra-Caballero, F. (2018). "Ciberactivismo y movimientos sociales. El espacio público oposicional en la tecnopolítica contemporánea”. Revista Latina de Comunicación Social, 73, 980 a 990. doi: 10.4185/RLCS-20181292

Slachevsky Aguilera, N. (2015). "Una revolución neoliberal: la política educacional en Chile desde la dictadura militar". Educação e Pesquisa, 41, 1473-1486.

Snowden, E. (2019). Permanent Record. New York City: Metropoli$\tan$ Books.

Soengas, X. (2013). "El papel de Internet y de las redes sociales en las revueltas árabes: una alternativa a la censura de la prensa oficial". Comunicar, 21 (41), 144-157. doi: 10.3916/C41-2013-14.

Soní-Soto, A. (2011). "Mitos y símbolos en la campaña de Barack Obama". Palabra Clave, 14 (1), 67-82.

Toal, G. (2009). “'In No Other Country on Earth': The Presidential Campaign of Barack Obama”. Geopolitics, 14 (2), 376-401. doi: 10.1080/14650040902828029.

Valenzuela, S., Arriagada, A.y Scherman, A. (2012). “The Social Media Basis of Youth Protest Behavior: The Case of Chile". Journal of Communication, 62 (2), 299-314. doi 10.1111/j.1460-2466.2012.01635.x.

Vesnic-Alujevic, L. (2012). "Political participation and web 2.0 in Europe: A case study of Facebook." Public Relations Review, 38(3), 466470. doi: 10.1016/j.pubrev.2012.01.010.

Walters, R. (2018). "How to tell a Fake: Fighting back against Fake News on the front lines of social media". Texas Review of Law \& Politics, 23 
MEDIOS SOCIALES Y DEMOCRATIZACIÓN DE LA COMUNICACIÓN: DEL POTENCIAL

(1), 111-179.

Wead, D. (2017). Game of Thorns: The Inside Story of Hillary Clinton's Failed Campaign and Donald Trump's Winning Strategy. New York City: Center Street.

Wring, D. y Ward, S. (2010). "The Media and the 2010 Campaign: the Television Election?” Parliamentary Affairs, 63 (4), 802-817. doi: 10.1093/ $\mathrm{pa} / \mathrm{gsq} 024$.

Zeynep, T. (2018). Twitter and Tear Gas: The Power and Fragility of Networked Protest. New Haven: Yale University Press. 